\title{
Presenting symptoms of giant fibrovascular polyp of the oesophagus: case report and literature review
}

\author{
S Sestini ${ }^{1}$, M Gisabella ${ }^{1}$, U Pastorino $^{1}$, A Billé ${ }^{2}$ \\ ${ }^{1}$ Fondazione IRCCS, Istituto Nazionale dei Tumori, Milan, Italy \\ ${ }^{2}$ Thoracic Surgery at Guys Hospital, UK
}

\section{ABSTRACT}

INTRODUCTION Lipomas of the gastrointestinal tract are rare, slow-growing lesions that comprise $0.4 \%$ of all gastrointestinal neoplasms. They can cause dysphagia, dyspnoea or sudden choking.

CASE HISTORY Due to rarity of this condition and its uncommon presentation, a literature review was carried out (PubMed). This search revealed 290 articles, of which 74 were considered pertinent and were evaluated. We report a case of a $13 \mathrm{~cm}$ pedunculated oesophageal lipoma that presented with increasing dysphagia and two episodes of suffocation. The patient underwent curative resection through a cervical approach.

CONCLUSIONS Resection is recommended for large $(>5 \mathrm{~cm})$ or symptomatic polyps. Outcomes are excellent given that lesions are universally benign and oesophageal resection is not required.

\section{KEYWORDS}

Oesophageal polyp - Dysphagia -Suffocation - Resection

Accepted 3 January 2016; published online XXX

\section{CORRESPONDENCE TO}

Andrea Billé, E: andrea_bille@hotmail.it

Oesophageal lipomas (OLs) are extremely rare, accounting for $4 \%$ of all benign oesophageal lesions, and fibrovascular polyps account for $<1 \% .^{1}$ More than $60 \%$ of OLs occur in the cervical and upper thoracic oesophagus, in the region of the cricopharyngeus muscle. They are usually solitary lesions seen more frequently in males. These lesions grow slowly and can be asymptomatic for many years. Patients start to complain of symptoms when these lesions are sufficiently large to cause dysphagia, dyspnoea or sudden choking. We describe a case of a pedunculated OL presenting with increasing dysphagia and two episodes of suffocation.

\section{Materials and Methods}

A literature review was undertaken via a PubMed search in February 2015. 'Hypopharynx' OR 'esophagus' OR 'esophageal' were combined with: 'polyp' OR 'polypoid' OR 'lipoma' OR 'fibroma' OR 'hamartoma' OR 'pedunculated' OR 'fibrovascular'. A total of 290 articles were retrieved. Seventy-four articles were considered to be pertinent and comprised individual case reports and small cases series (maximum of 4 patients). Lesions ranged in diameter from $7 \mathrm{~mm}$ to $30 \mathrm{~cm}$ (mean, $70 \mathrm{~mm}$ ). Symptoms were present in $58(78.4 \%)$ patients whereas $13(17.5 \%)$ had no symptoms in the upper airways or gastrointestinal system. In 24 cases $(32.4 \%)$, the lesion was removed by endoscopic means. In $42(56.7 \%)$ cases the lesion was resected, whereas in $4(5.4 \%)$ cases an intervention was not needed. Also, in 4 cases, the intervention was not specified.

\section{Case History}

A 70-year old male presented with a history of increasing dysphagia during the previous 2 years. The patient described two episodes of sudden choking, the latter being immediately before hospital admission in March 2014. In May 2013, computed tomography (CT) of the neck and chest showed a cyst in the left pyriform sinus. Due to worsening symptoms, an oesophagoscopy was carried out in December 2013: it showed a normal mucosa, and what was misinterpreted as a small cystic lesion of the pyriform sinus. However, a biopsy at this site resulted in the diagnosis of an oesophageal angiolipoma. Magnetic resonance imaging (MRI) and CT showed a $13 \mathrm{~cm}$ lesion with a $3 \mathrm{~cm}$ pedicle in the cricopharyngeal muscle extending down to the mid-thoracic oesophagus (Fig 1a-b). Positron emission tomography integrated with computed tomography (PETCT) did not show pathological uptake, with the exception of the distal end of the polyp (maximum standardised uptake $=6$, Fig 1c). The patient was discussed at the 


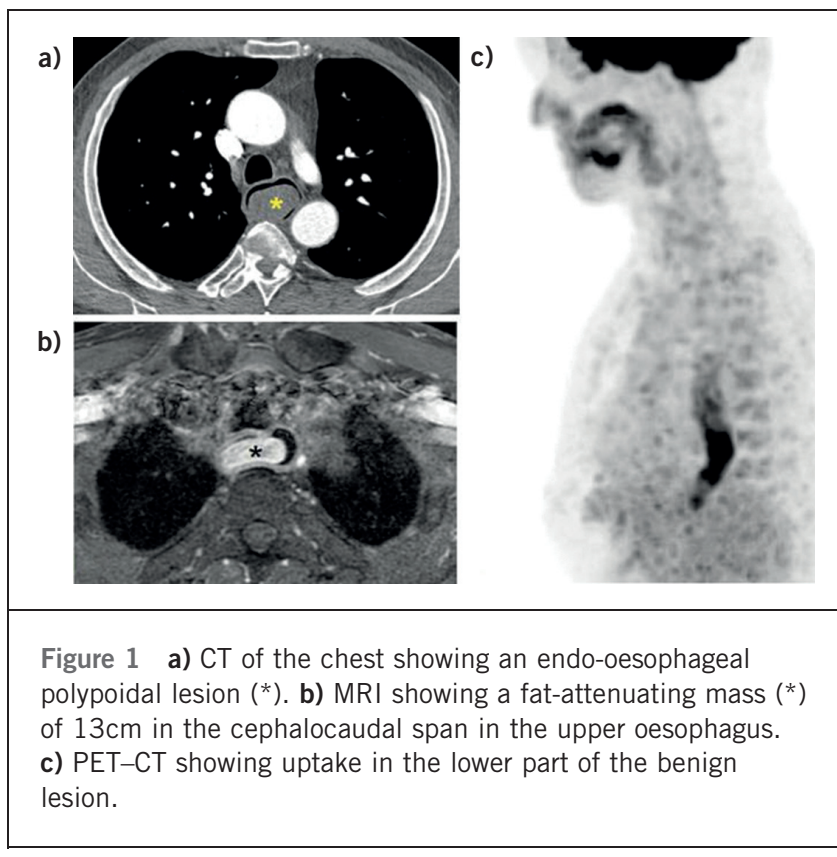

multidisciplinary meeting and, due to the high uptake and dimension of the polyp, resection was recommended.

At the time of surgery, oesophagoscopy under general anaesthesia revealed a hyperplastic and corrugated oesophageal mucosa without any abnormality. A right cervicotomy was done along the right sternocleidomastoid muscle. After isolation of the right recurrent nerve, the oesophagus was opened (Fig 2a). A 13cm polyp was extracted from the oesophagus (Fig 2b), and there was no evidence of invasion of the oesophageal wall. The pedicle was isolated and stapled, and the polyp removed completely.

At the end of the procedure but before waking-up the patient, an upper gastrointestinal endoscopy was done: the stapler line and absence of a stricture of the oesophagus were noted. A nasogastric tube was placed and the patient made an uneventful recovery.

Histology revealed a $15 \mathrm{~cm}$ mass with a smooth surface originating from the left wall of the oesophagus, in the area of the cricopharyngeus muscle. The distal end of the polyp displayed a focus of ulceration, and was the cause of a positive result on PET.

\section{Discussion}

OLs or fibrovascular polyps are rare. Symptoms are directly correlated with polyp size. Typically, patients with large polyps present with dysphagia and a sense of 'fullness' in the throat. Partial displacement of the polyp into the mouth is a common event. However, the most severe and life-threatening complication is complete regurgitation of the tumour, and sudden death due to asphyxiation.

Typically, these tumours are $2-4 \mathrm{~cm}$ in diameter at the base, of length $\leq 25 \mathrm{~cm}$, with a 'sausage' shape and thin upper pedicle; ulceration is usually present at the distal

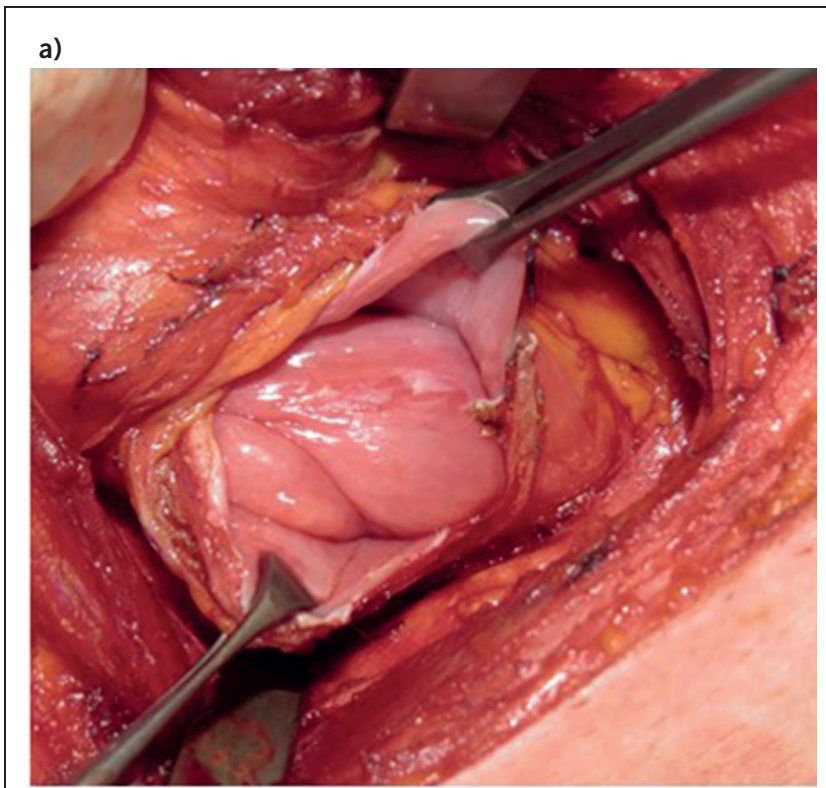

b)

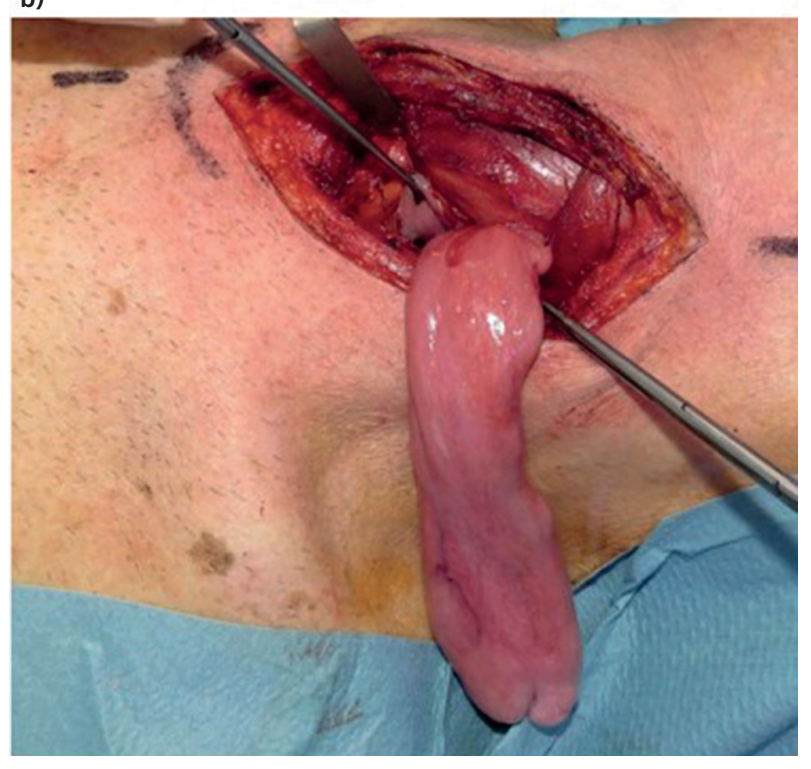

Figure 2 a) A $13 \mathrm{~cm}$ polyp within the oesophagus. b) The polyp (covered with normal mucosa) is extracted from the oesophagus.

end. $^{2}$ Ten cases of sudden death have been reported. ${ }^{3}$ These lesions can be misdiagnosed for years because the polyp is usually covered by normal mucosa (as in our patient).

CT and MRI can provide the correct diagnosis by evaluation of the adipose component in tissue and infiltration of the oesophageal wall. Malignant degeneration is rare, but resection is first-line treatment and cannot be delayed for large oesophageal polyps due to the risk of regurgitation and asphyxia. 
The surgical approach (cervicotomy, thoracotomy, videothoracoscopy or endoscopy) is dependent upon tumour size and pedicle location. Asymptomatic lipomas of the gastrointestinal tract $<1 \mathrm{~cm}$ in diameter do not cause symptoms and do not require specific treatment. ${ }^{2,4}$ Small pedunculated polyps $(<2 \mathrm{~cm}$ in diameter with a thin pedicle) can be resected by endoscopic means. ${ }^{4}$ In the case of large $(>5 \mathrm{~cm})$ symptomatic polyps, or those with a large vascularised pedicle, resection via a transcervical (or, rarely, a transthoracic) approach is advocated due to the difficulties in removal of a large lesion and haemorrhage risk. A combined approach with pharyngotomy and gastrostomy is recommended for polyps that extend into the stomach and have a bulbous tip distal to the stomach. ${ }^{3,5}$

\section{Conclusions}

Large, benign fibrovascular polyps of the oesophagus can cause life-threatening complications because of their unusual and misleading presentation. The correct diagnosis and urgent surgical or endoscopic resection (which are dependent upon the size and location of the lesion) are essential to prevent sudden asphyxia and death. Our surgical team recommend an open surgical approach for polyps of diameter $>5 \mathrm{~cm}$.

\section{References}

1. Wu MH, Chuang CM, Tseng YL. Giant intraluminal polyp of the esophagus. Hepatogastroenterology 1998; 45: 2,115-2,116.

2. Feldman J, Tejerina M, Hallowell M. Esophageal lipoma: a rare tumor. J Radiol Case Rep 2012; 6: 17-22.

3. Lecleire S, Di Fiore F, Roque I et al. Asphyxia due to a laryngeal lipoma following esophageal endosonography. Endoscopy 2003; 35: 254.

4. Hong JB, Choi CW, Kim HW et al. Endoscopic resection using band ligation for esophageal SMT in less than $10 \mathrm{~mm}$. World J Gastroenterol 2015; 21: 2,982-2,987.

5. Carrisk C, Collins KA, Lee CJ et al. Sudden death due to asphyxia by esophageal polyp. Am J Forensic Med Pathol 2005; 26: 275-281. 